\title{
Dense molecular gas around AGN: HCN/CO in NGC $3227^{\star}$ (Research Note)
}

\author{
R. Davies ${ }^{1}$, D. Mark ${ }^{2}$, and A. Sternberg ${ }^{2}$ \\ 1 Max Planck Institut für extraterrestrische Physik, Postfach 1312, 85741 Garching, Germany \\ e-mail: davies@mpe.mpg.de \\ 2 Sackler School of Physics and Astronomy, Tel Aviv University, Tel Aviv 69978, Israel \\ Received 7 July 2011 / Accepted 15 November 2011
}

\section{ABSTRACT}

\begin{abstract}
There is now convincing evidence that the intensity of $\mathrm{HCN}$ molecular line emission is enhanced around active galactic nuclei. In this paper we examine the specific case of the Seyfert galaxy NGC 3227, for which there are subarcsecond resolution data for the HCN (1-0) $88 \mathrm{GHz}$ and $\mathrm{CO}(2-1) 230 \mathrm{GHz}$ rotational lines, enabling us to spatially separate a circumnuclear ring at a radius of $140 \mathrm{pc}$ and an inner nuclear region within $40 \mathrm{pc}$ of the $\mathrm{AGN}$. The $\mathrm{HCN}(1-0) / \mathrm{CO}(2-1)$ flux ratio differs by more than an order of magnitude between these two regions. We carry out large velocity gradient (LVG) computations to determine the range of parameters (gas temperature and density, $\mathrm{HCN} / \mathrm{CO}$ abundance ratio, column density, and velocity gradient) that yield physically plausible solutions for the observed flux ratio in the central $100 \mathrm{pc}$. The observed $\mathrm{HCN} / \mathrm{CO}$ intensity ratio in the nucleus is consistent with very optically thick thermalized emission in very dense $\left(\gtrsim 10^{5} \mathrm{~cm}^{-3}\right)$ gas, in which case the $\mathrm{HCN} / \mathrm{CO}$ abundance ratio there is unconstrained. Alternatively, the $\mathrm{HCN} / \mathrm{CO}$ intensity ratio could be due to optically thinner emission but from gas with a very high $\left(\sim 10^{-2}\right) \mathrm{HCN} / \mathrm{CO}$ abundance ratio. This possibility is more consistent with the $\mathrm{CO}$ and $\mathrm{HCN}$ emission seen in the nuclei of the Seyfert galaxies NGC 1068 and NGC 6951. It would imply the velocity gradients are large and the clouds may be gravitationally unbound. We estimate that the X-ray ionisation rate at radii less than $20 \mathrm{pc}$ in the centre of NGC 3227 exceeds $10^{-13} \mathrm{~s}^{-1}$. X-ray ionisation and heating may lead to high $\mathrm{HCN} / \mathrm{CO}$ ratios in warm gas in a high-ionisation molecular phase near the AGN.
\end{abstract}

Key words. galaxies: active - galaxies: individual: NGC 3227 - galaxies: ISM - galaxies: nuclei - radio lines: galaxies

\section{Introduction}

During the past decade, there has been an increasing observational effort to understand the nature of the HCN emission from galaxies. This has included studies of $\mathrm{HCN}$ emission in nearby AGN and starbursts (Kohno et al. 2001; Kohno 2005; Kohno et al. 2008; Krips et al. 2007, 2008), as well as luminous and ultraluminous galaxies (Graciá-Carpio et al. 2006, 2008). One result is that $\mathrm{HCN}$ line emission is enhanced with respect to $\mathrm{CO}$ in at least some AGN compared with starbursts (Kohno 2005; Kohno et al. 2008). These high HCN/CO intensity ratios could be due to high molecular gas densities near the AGN, and/or high $\mathrm{HCN} / \mathrm{CO}$ abundance ratios that might be a result of elevated X-ray ionisation and heating rates near the accreting black holes. For example, in an early study, Sternberg et al. (1994) concluded that the large $\mathrm{HCN} / \mathrm{CO}$ intensity ratio observed in the nuclear $(<100 \mathrm{pc})$ region in the Seyfert-2 galaxy NGC 1068 indicates a high HCN/CO abundance ratio $\sim 10^{-2}$ near the AGN. Lepp \& Dalgarno (1996) and Usero et al. (2004) argued that elevated $\mathrm{HCN} / \mathrm{CO}$ abundance ratios are signatures of high X-ray ionisation rates (Maloney et al. 1996; Meijerink \& Spaans 2005; Boger \& Sternberg 2005; Meijerink et al. 2007). High gas densities also likely play a role in boosting the $\mathrm{HCN}$ line intensity (Krips et al. 2008).

In this paper we present high resolution observations of $\mathrm{CO}$ (2-1) and HCN (1-0) rotational line emissions in the inner regions of NGC 3227, a nearby $\left(D=17 \mathrm{Mpc} ; 1^{\prime \prime} \sim 80 \mathrm{pc}\right)$ Seyfert

\footnotetext{
* Based on observations carried out with the IRAM Plateau de Bure Interferometer. IRAM is supported by INSU/CNRS (France), MPG (Germany) and IGN (Spain).
}

galaxy for which detailed studies have been made of the stellar (Davies et al. 2006, 2007) and gaseous (Schinnerer et al. 2000; Hicks et al. 2009) content of its central regions. We analyze the data using large-velocity-gradient (LVG) computations. For this galaxy, we again find a significant enhancement in the $\mathrm{HCN} / \mathrm{CO}$ intensity ratio close to the active nucleus. The observed nuclear intensity ratio is consistent with optically thick thermalized emission in dense $\left(\gtrsim 10^{5} \mathrm{~cm}^{-3}\right)$ gas. Alternatively, and especially in comparison with similar data for NGC 1068 and NGC 6951, the nuclear emissions could be tracing optically thinner emission in which the $\mathrm{HCN} / \mathrm{CO}$ abundance ratio is large.

\section{Observations}

The analysis in this paper is based on subarcsecond resolution observations of the $\mathrm{CO}(2-1) 230.5 \mathrm{GHz}$ and $\mathrm{HCN}(1-0)$ $88.6 \mathrm{GHz}$ lines. The $\mathrm{CO}(2-1)$ data, for which the beam is $0.6^{\prime \prime}$, were previously presented by Schinnerer et al. (2000). New $3 \mathrm{~mm} \operatorname{HCN}(1-0)$ data, with a $0.9^{\prime \prime} \times 1.2^{\prime \prime}$ beam, were obtained during February 2009 in the A configuration $(760 \mathrm{~m}$ baseline) of the six $15-\mathrm{m}$ antennas of the IRAM Plateau de Bure Interferometer. The $\mathrm{H}^{12} \mathrm{CN}(1-0)$ line at $88.6 \mathrm{GHz}$ and the $\mathrm{H}^{13} \mathrm{CN}(1-0)$ line at $86.3 \mathrm{GHz}$ were observed together, using a single polarisation for each $1 \mathrm{GHz}$ bandwidth segment. The system temperature was $80-100 \mathrm{~K}$. Atmospheric conditions were moderate, with winds and $\sim 5 \mathrm{~mm}$ precipitable water vapour. Phase and amplitude variations were calibrated out by interleaving reference observations of standard calibration sources. The data were processed and calibrated using the CLIC program in the IRAM GILDAS package, and binned spectrally to a 


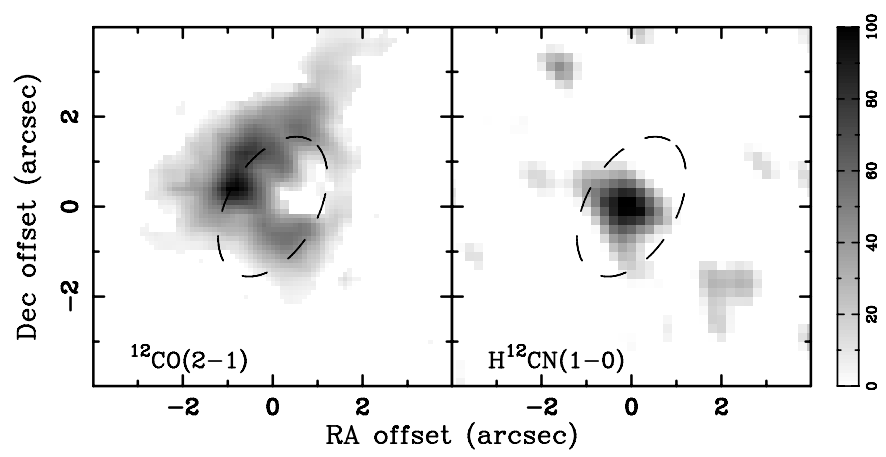

Fig. 1. Images of the $\mathrm{CO}(2-1)$ line emission (from Schinnerer et al. $2000)$ and $\mathrm{HCN}(1-0)$ line emission from NGC 3227. Superimposed is the ellipse tracing the circumnuclear ring (Davies et al. 2006). While the $\mathrm{CO}$ emission originates mostly from the ring, the $\mathrm{HCN}$ intensity is far higher in the central region. There is an order-of-magnitude change in the flux ratio between these regions.

Table 1. CO and HCN fluxes in NGC 3227.

\begin{tabular}{lcrc}
\hline \hline aperture & $\begin{array}{c}F_{\mathrm{HCN} 1-0} \\
\mathrm{Jy} \mathrm{km} \mathrm{s}^{-1}\end{array}$ & $\begin{array}{c}F_{\mathrm{CO} 2-{ }^{a}}{ }^{a} \\
\mathrm{Jy} \mathrm{km} \mathrm{s}^{-1}\end{array}$ & $F_{\mathrm{HCN} 1-0} / F_{\mathrm{CO} 2-1}$ \\
\hline $1^{\prime \prime}$ & $0.48 \pm 0.06$ & $4.4 \pm 1.3$ & $0.114_{-0.035}^{+0.051}$ \\
$2^{\prime \prime}$ & $1.37 \pm 0.17$ & $19.5 \pm 5.9$ & $0.074_{-0.023}^{+0.033}$ \\
$4^{\prime \prime}$ & $1.86 \pm 0.29$ & $57.6 \pm 17.3$ & $0.033_{-0.011}^{+0.016}$ \\
$2-4^{\prime \prime}$ & $0.49 \pm 0.33$ & $38.5 \pm 18.3$ & $0.013_{-0.008}^{+0.024}$ \\
\hline
\end{tabular}

Notes. ${ }^{(a)}$ Scaled from the $75 \mathrm{mJy}$ given by Schinnerer et al. (2000) in a $8.4^{\prime \prime} \times 8.4^{\prime \prime}$ aperture and with an uncertainty of $30 \%$.

resolution of $50 \mathrm{~km}^{-1}$ to increase the signal-to-noise ratio. The kinematics of the HCN(1-0) line emission in NGC 3227 are analysed by Sani et al. (2011) together with data for several other galaxies.

It is remarkable that, despite the similar beam sizes, the $\mathrm{CO}(2-1)$ map clearly shows the circumnuclear ring at a radius of $140 \mathrm{pc}\left(1.7^{\prime \prime}\right)$, while the HCN map shows emission from only the central region, within a few tens of parsecs from the nucleus. This can be seen clearly in Fig. 1 and is reflected in the line fluxes given in Table. 1 . In the nuclear region, which we define here to be the central $1^{\prime \prime}$ (i.e. $r<40 \mathrm{pc}$ ), the flux ratio is $F_{\mathrm{HCN} 1-0} / F_{\mathrm{CO} 2-1}=0.11$. In the circumnuclear ring, for which we take an annulus $2-4$ ", we find that $F_{\mathrm{HCN} 1-0} / F_{\mathrm{CO} 2-1}=0.01$, which is an order of magnitude less. Since the emission is spatially resolved in both cases, this difference cannot be due to differences in the beam dilution effects. Instead, it is likely to be caused by differences in either molecular abundance - e.g., as a result of a distance dependent X-ray ionisation rate - or differences in excitation efficiencies - e.g., owing to higher nuclear gas densities favouring $\mathrm{HCN}$ emission. (The critical $\mathrm{H}_{2}$ density of $\sim 7 \times 10^{3} \mathrm{~cm}^{-3}$ for collisional deexcitation of $\mathrm{CO}(2-1)$ is significantly lower that the $\sim 3 \times 10^{6} \mathrm{~cm}^{-3}$ for $\mathrm{HCN}(1-0)$.) In the next section we use LVG models to address these issues.

\section{Physical properties of the molecular gas}

\subsection{LVG calculations}

We have constructed a new LVG code and used it to compute the $\mathrm{HCN}(1-0)$ and $\mathrm{CO}(2-1)$ line intensities for a wide range of physical conditions. We calculated the line source functions assuming photon escape probabilities from spherical clouds. We used the recent data from Yang et al. (2010) for the excitations and deexcitations of the $\mathrm{CO}$ rotational levels that are induced by collisions with $\mathrm{H}_{2}$. For $\mathrm{HCN}$ we used the Green \& Thaddeus (1974) collisional data, as updated and listed in the RADEX database (Van der Tak et al. 2007). We have cross-checked all of our results with the LVG code RADEX, and found excellent agreement: in the parameter space that we assessed, the converged line ratios agree to within $1.5 \%$. Here we assume that the $\mathrm{HCN}$ and $\mathrm{CO}$ molecules are mixed uniformly, and that the corresponding line emissions originate in gas at the same temperature and density. The luminosity ratios resulting from the model calculations are presented graphically in Fig. 2, covering the following parameter space: kinetic temperature $30 \leq T[K] \leq 300$, $\mathrm{HCN}$ to $\mathrm{CO}$ abundance ratio $10^{-5} \leq X_{\mathrm{HCN}} / X_{\mathrm{CO}} \leq 10^{-2}$ (with $X_{\mathrm{CO}}=10^{-4}$ as the $\mathrm{CO}$ abundance relative to hydrogen), $\mathrm{H}_{2}$ volume gas density $10^{3} \leq n_{\mathrm{H}_{2}}\left[\mathrm{~cm}^{-3}\right] \leq 10^{7}$, and a ratio of gasdensity to velocity-gradient, or equivalently column density to linewidth, of $5 \times 10^{17} \leq N_{\mathrm{H}_{2}} / \mathrm{d} V\left[\mathrm{~cm}^{-2}\left(\mathrm{~km} \mathrm{~s}^{-1}\right)^{-1}\right] \leq 5 \times 10^{23}$.

In each panel of Fig. 2, the parameter-space consists of four regimes. The upper right part of each panel corresponds to local thermal equilibrium (LTE) in the optically thick limit. In this regime, the transition excitation temperatures $T_{\text {ex }}$ of the lines approaches the kinetic temperature $T_{\text {kin }}$ of the gas. Since the line flux, in the Rayleigh-Jeans limit, is $F \propto \int T_{\mathrm{ex}} v^{2} \mathrm{~d} v$, and an interval $\mathrm{d} V$ in velocity space is $\mathrm{d} V=c \mathrm{~d} v / v$, the ratio of two line fluxes measured as $\int F \mathrm{~d} V$ (e.g. in units of $\mathrm{Jy} \mathrm{km} \mathrm{s}^{-1}$ as used here) is $F_{1} / F_{2}=\left(v_{1} / v_{2}\right)^{2}$ in the optically thick and LTE limit. This corresponds to 0.15 for the ratio of the $\mathrm{HCN}(1-0)$ and $\mathrm{CO}(2-1)$ lines at $88.6 \mathrm{GHz}$ and $230.5 \mathrm{GHz}$ respectively, which is close to the observed ratio of 0.1 in the nucleus.

The upper left regions also correspond to LTE because the gas densities are high, but in this regime the line optical depths are low because the velocity gradients (or line widths) are large. For these conditions, the line intensities are linearly proportional to the molecular abundances. This behaviour is reflected in the $\mathrm{HCN} / \mathrm{CO}$ intensity ratios indicated by the contour values. In the left sides of the panels, the intensity ratios decrease linearly with the assumed abundance ratio $X_{\mathrm{HCN}} / X_{\mathrm{CO}}$, which ranges from $10^{-2}$ to $10^{-5}$ in Fig. 2.

The lower parts of each panel correspond to low densities for which the HCN is subthermally excited, leading to relatively higher populations in the lowest rotational levels. Again the optical depth increases from left to right as the column density increases for fixed line width.

Some regions of the parameter space may be less physically plausible because they assume very large velocity gradients. Lines of constant velocity gradient are indicated by the dashed orange lines in Fig. 2, with increasing gradients towards the upper left. For self-gravitating virialised clouds $\Delta V / R \sim n^{1 / 2}$ where $\Delta V$ is the velocity dispersion, $R$ is the cloud radius, and $n$ is the gas density. Treating $\Delta V / R$ as a velocity gradient (e.g. Goldsmith 2001) gives $\mathrm{d} V / \mathrm{d} r \sim 3.1 \mathrm{~km} \mathrm{~s}^{-1} \mathrm{pc}^{-1} \sqrt{n_{\mathrm{H}_{2}} / 10^{4} \mathrm{~cm}^{-3}}$, or typically a few $\mathrm{km} \mathrm{s}^{-1} \mathrm{pc}^{-1}$, or a few tens in cases of extreme density. In Fig. 2, the virial relation $n_{\mathrm{H}_{2}} \propto\left(N_{\mathrm{H}_{2}} / \mathrm{d} V\right)^{2}$ is represented by the dot-dash blue line in each panel. To the left of this line, one might find clouds that are unbound or at least partially pressure confined.

\subsection{Analysis for NGC 3227}

In Fig. 2, we have superimposed curves representing the possible parameter ranges for the measured flux ratios $F_{\mathrm{HCN} 1-0} / F_{\mathrm{CO} 2-1}$ of NGC 3227 for the nucleus (red lines) and circumnuclear ring 

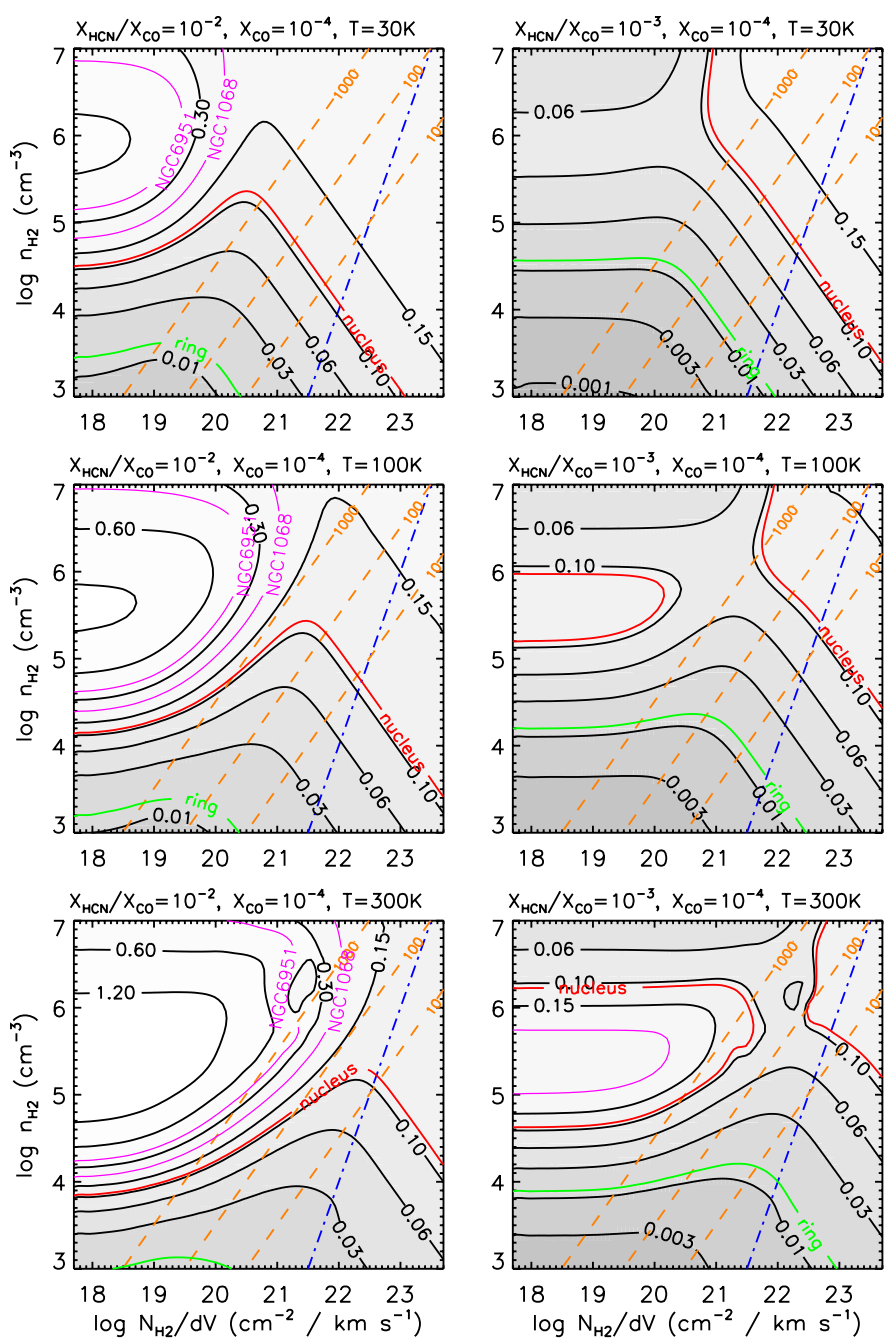
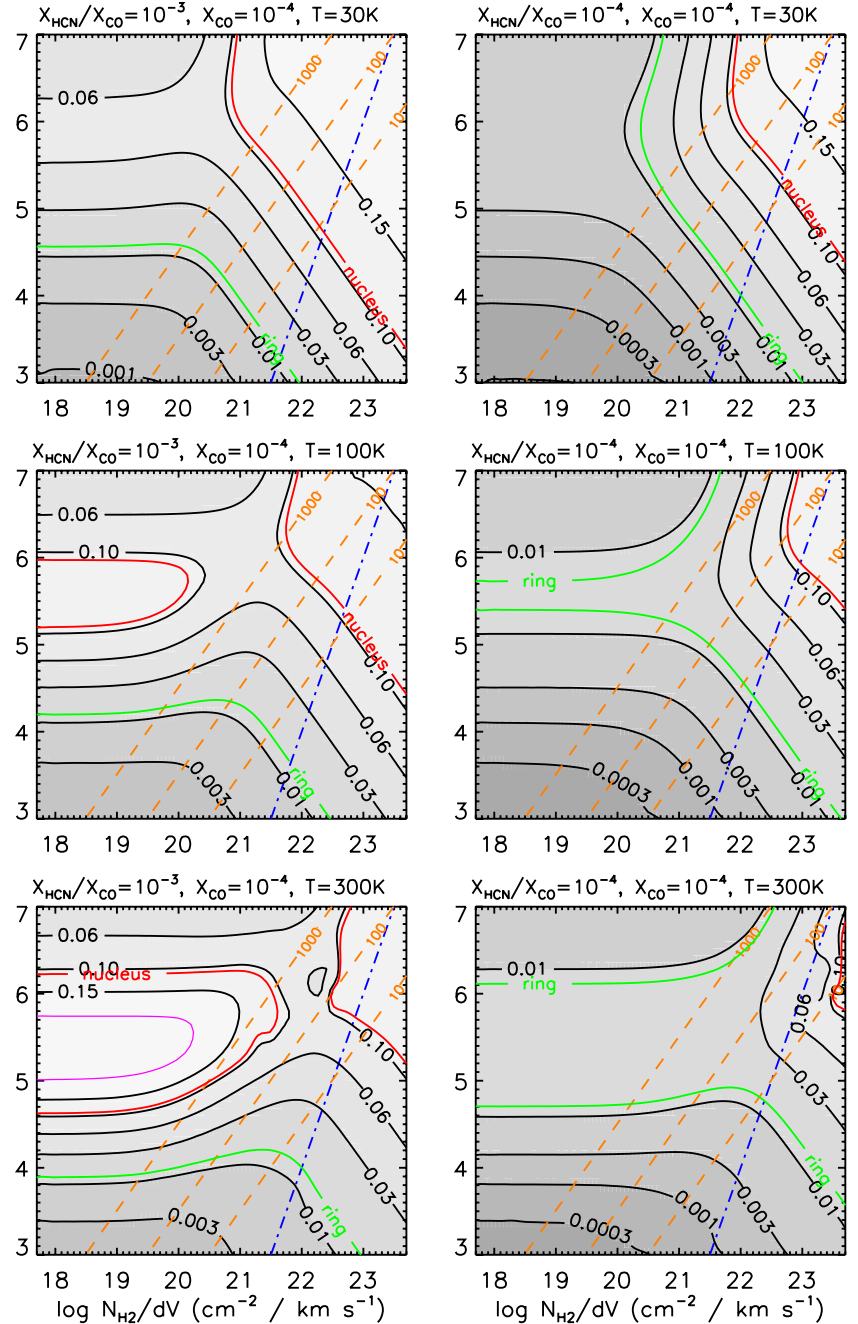
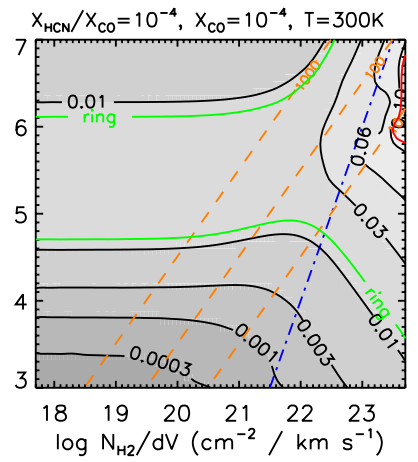

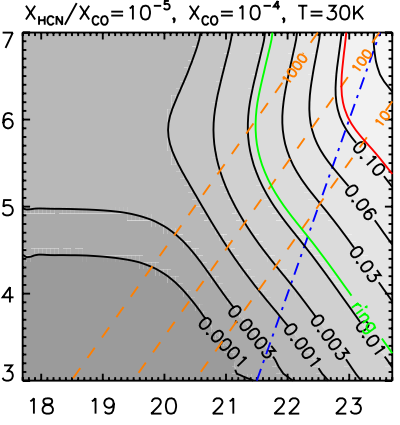

$X_{\mathrm{HCN}} / \mathrm{X}_{\mathrm{CO}}=10^{-5}, \mathrm{X}_{\mathrm{CO}}=10^{-4}, \mathrm{~T}=100 \mathrm{~K}$
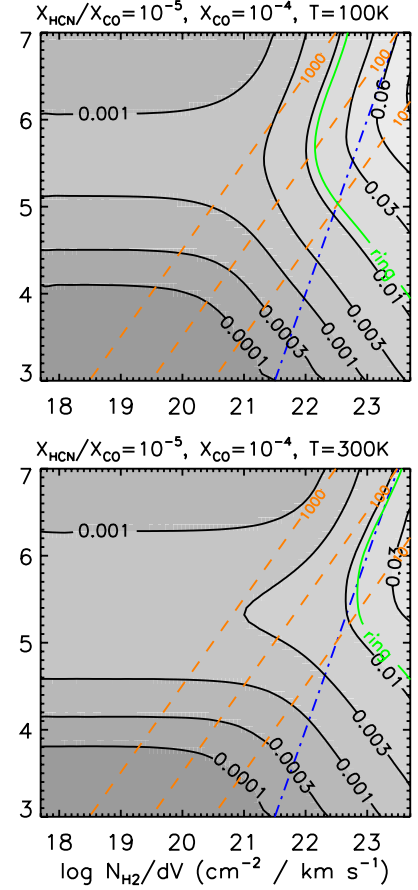

Fig. 2. LVG calculations for a four-dimensional parameter space: the $[\mathrm{HCN}] /[\mathrm{CO}]$ abundance ratio and gas kinetic temperature are given at the top of each panel, while the axes of each panel are the gas volume density and the ratio of column density to linewidth (or equivalently of volume density to velocity gradient). The contours show the expected line ratio based on the emitted fluxes in Jy km s${ }^{-1}$. The locus representing the range of parameters possible for the nucleus and ring of NGC 3227 are drawn in red and green respectively. The dashed orange lines are curves of equal velocity gradient (in $\mathrm{km} \mathrm{s}^{-1} \mathrm{pc}^{-1}$ ), and indicate which regions of the parameter space are physically plausible. In particular, the locus of self-gravitating virialised clouds is represented by the dot-dash blue line.

(green lines), as defined in Sect. 2. If we assume that the clouds are self-gravitating, we are restricted to the points where the red and green lines intersect the dashed blue line. The curves show that if the clouds in the ring have a similar density to the nucleus, then the HCN abundance must be about two orders of magnitude lower. Alternatively, the density may differ by up to two orders of magnitude if the abundances are similar. We discuss this further in Sect. 3.4.

In the nucleus, the flux ratio $F_{\mathrm{HCN} 1-0} / F_{\mathrm{CO} 2-1}=0.11$ is remarkably close to the LTE optically thick limit, and its locus includes a contour around this region. However, depending on the $\mathrm{HCN}$ abundance, the gas density at which this occurs can vary from $n_{\mathrm{H}_{2}} \sim 10^{4} \mathrm{~cm}^{-3}$ at the highest abundance to $\sim 3 \times 10^{5} \mathrm{~cm}^{-3}$ at the lowest abundance we have considered, with column density $N_{\mathrm{H}_{2}} / \mathrm{d} V \gtrsim 10^{22} \mathrm{~cm}^{-2} /\left(\mathrm{km} \mathrm{s}^{-1}\right)$. By considering the mean volume density of the $\mathrm{HCN}$ emitting region, and putting a limit on a realistic filling factor, we can restrict this range further.

The first step is to estimate the volume of the emitting region. This can be done because the HCN emission is marginally resolved. A detailed estimate of the intrinsic size - via dynamical modelling, and taking into account emission from the ring - is given in Sani et al. (2011). These authors show that the diameter is $0.54^{\prime \prime}$, corresponding to $45 \mathrm{pc}$, and that the scale height is $6 \mathrm{pc}$. Taking this as an indication of the size along the line of sight sets the volume of the emitting region.

The second step is to estimate the mass. We have done this in several ways because they are all uncertain.

1. The LVG calculation directly yields a mass under the assumption that the ratio of the observed linewidth to the linewidth of an individual cloud traces the number of clouds, such that $N_{\text {tot }}=N_{\text {cloud }}\left(\delta v_{\text {obs }} / \delta v_{\text {cloud }}\right)$. For conditions corresponding to $X_{\mathrm{HCN}} / X_{\mathrm{CO}}=10^{-2}, T=300 \mathrm{~K}$, $n_{\mathrm{H}_{2}}=10^{5.5} \mathrm{~cm}^{-3}$, and $N_{\mathrm{H}_{2}} / \mathrm{d} V=10^{22} \mathrm{~cm}^{-2}\left(\mathrm{~km} \mathrm{~s}^{-1}\right)^{-1}$ (see Sect. 3.3), the observed HCN(1-0) flux leads to a mass of $1.8 \times 10^{6} M_{\odot}$.

2. The standard method for estimating the mass is based on the $\mathrm{CO}$ luminosity. We applied this directly to the $\mathrm{CO}(2-1)$ line flux in Table 1 using a conversion factor $\alpha=4.3$, which includes a correction for helium (Tacconi et al. 2008). It yields $3.3 \times 10^{6} M_{\odot}$. 

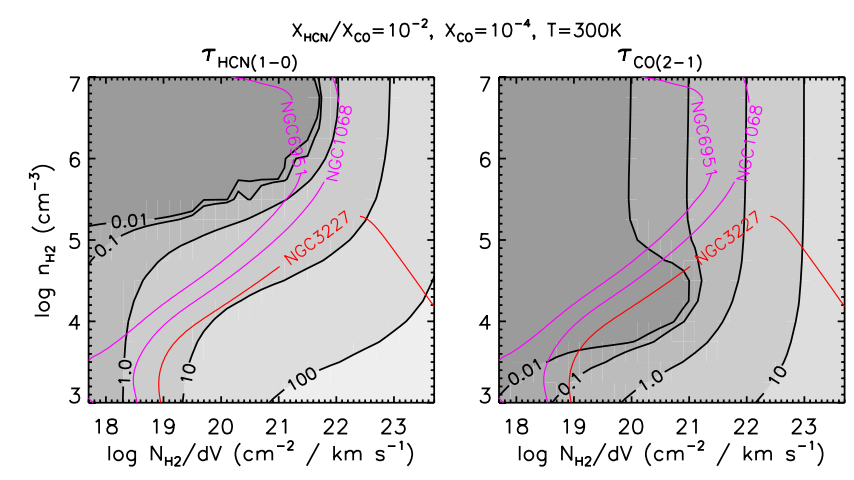

Fig. 3. Optical depth $\tau$ of the $\mathrm{HCN}(1-0)$ line (left) and $\mathrm{CO}(2-1)$ line (right) for gas properties corresponding to the bottom left panel in Fig. 2. Darker regions correspond to lower optical depth. We superimpose contours for the three galaxies corresponding to their $\mathrm{HCN}(1-0) / \mathrm{CO}(2-1)$ ratio.

3. A similar conversion for the HCN line, that has been calibrated by Krips et al. (2008) for AGN, is $M_{\mathrm{H}_{2}} / L_{\mathrm{HCN}} \sim 10 M_{\odot}$ $\left(\mathrm{K} \mathrm{km} \mathrm{s}^{-1} \mathrm{pc}^{2}\right)^{-1}$. This yields a mass of $6 \times 10^{6} M_{\odot}$.

4. As a final check, we used the dynamical mass derived from the HCN kinematics. By fitting models to account for the beam smearing, Sani et al. (2011) found that $M_{\text {dyn }}=5.6 \times$ $10^{7} M_{\odot}$. For a nominal $10 \%$ gas fraction expected in local discs and starbursts (Hicks et al. 2009), this would yield a gas mass of $6 \times 10^{6} M_{\odot}$.

These estimates are all of the same order of magnitude, and suggest that the gas mass in the central arcsec is of the order of $4 \times 10^{6} M_{\odot}$. Hence we can estimate the mean density to be $\left\langle n_{\mathrm{H}_{2}}\right\rangle \gtrsim 6 \times 10^{3} \mathrm{~cm}^{-3}$. Comparing this to the cloud densities above yields volume filling factors in the range $1-0.01$. In this range, a lower filling factor is more physically plausible, and would tend to favour solutions with higher cloud densities. Figure 2 shows these solutions have either higher temperature or less extreme $\mathrm{HCN}$ abundance.

\subsection{Comparison to NGC 6951 and NGC 1068}

Two other galaxies for which the $\mathrm{HCN}(1-0) / \mathrm{CO}(2-1)$ ratio has been measured on comparable $\sim 100$ pc scales are NGC 1068 and NGC 6951. We use flux densities reported by Krips et al. (2007) for the nuclear region (denoted "C" in their Table 1) of NGC 6951. For the circumnuclear disc of NGC 1068, we use the sum of the red and blue channels reported in Table 3 of Usero et al. (2004). These yield line ratios (for line fluxes in $\mathrm{Jy} \mathrm{km} \mathrm{s}^{-1}$ ) of $0.37 \pm 0.05$ and $0.214 \pm 0.002$ respectively, and are denoted by the solid magenta lines in Fig. 2. These lines appear almost exclusively in the panels corresponding to the highest $\mathrm{HCN}$ abundance we have considered, $X_{\mathrm{HCN}} / X_{\mathrm{CO}}=10^{-2}$.

In contrast to NGC 3227 , in which the line emission appears to be optically thick, the loci of the magenta lines for NGC 1068 and NGC 6951 are closer to the optically thin (left) side of the panels. Despite this, we note that there are regions in the parameter space where the contours corresponding to all three objects lie close together, running from lower left to upper right. The region extends from $n_{\mathrm{H}_{2}}=10^{4} \mathrm{~cm}^{-3}$ and $N_{\mathrm{H}_{2}} / \mathrm{dV}=$ $10^{19} \mathrm{~cm}^{-2} /\left(\mathrm{km} \mathrm{s}^{-1}\right)$ to $n_{\mathrm{H}_{2}}=10^{6} \mathrm{~cm}^{-3}$. It is precisely because one can attribute the observed line ratios - with different optical depths for the three galaxies - to similar physical properties of the gas in all these three objects that this locus is appealing.
The reason why this region exists can be seen in Fig. 3, which shows the optical depths $\tau$ for the $\mathrm{HCN}(1-0)$ and $\mathrm{CO}(2-1)$ transitions. The gas properties of both these panels correspond to the bottom left panel in Fig. $2\left(300 \mathrm{~K}\right.$ and $\left.X_{\mathrm{HCN}} / X_{\mathrm{CO}}=10^{-2}\right)$, and cover the same range of density and velocity gradient. These plots clearly show the characteristics of the different regions: in the lower half, the $\mathrm{HCN}(1-0)$ line is optically thick because the density is low enough that it is sub-thermal; above the critical density, the line is in LTE and thus optically thin at low columns and optically thick at high columns. The locus where all the contours for the three galaxies are close together and parallel approximately follows the boundary where the $\mathrm{HCN}(1-0)$ line becomes optically thick. Here, a small change in physical conditions (column or density) can result in the $\mathrm{HCN}(1-0)$ emission switching from optically thin to optically thick.

This regime is, however, also associated with very large velocity gradients. They range from $\mathrm{d} V / \mathrm{d} r \sim 10^{4} \mathrm{~km} \mathrm{~s}^{-1} \mathrm{pc}^{-1}$ at $T=30 \mathrm{~K}$, decreasing as the temperature increases. Velocity gradients were not discussed explicitly by either Sternberg et al. (1994) or Usero et al. (2004) in their $T=50 \mathrm{~K} \mathrm{LVG}$ calculations for NGC 1068. Nevertheless their analyses also associate the observed properties with similarly extreme velocity gradients. Indeed, one of the main conclusions of Sternberg et al. (1994) was that $X_{\mathrm{HCN}} / X_{\mathrm{CO}} \gtrsim 10^{-2}$ in NGC1068. For the temperature that they considered, this would lead to $\mathrm{d} V / \mathrm{d} r \sim 10^{4} \mathrm{~km} \mathrm{~s}^{-1} \mathrm{pc}^{-1}$ (matching the top left panel of Fig. 2 here). However, our LVG calculations shows that $\mathrm{d} V / \mathrm{d} r$ is reduced as both the temperature and density increase. When considering all three galaxies together, the smallest - and therefore arguably the most physically plausible - value in the parameter space we have covered is $\mathrm{d} V / \mathrm{d} r \sim 100 \mathrm{~km} \mathrm{~s}^{-1} \mathrm{pc}^{-1}$ at $T=300 \mathrm{~K}$ and $n_{\mathrm{H}_{2}} \sim 10^{5.5} \mathrm{~cm}^{-3}$. This location is not far from the boundary of the optically thick LTE regime discussed previously, but owing to the high velocity gradient represents clouds that are either pressure confined or unbound.

Interestingly, there is evidence in NGC 1068 from recent Herschel observations with PACS of high rotational CO transitions, for a significant mass of molecular gas in the central $\sim 100 \mathrm{pc}$ at temperatures of $140 \mathrm{~K}$ and $430 \mathrm{~K}$ and densities of $\sim 10^{6} \mathrm{~cm}^{-3}$ (Hailey-Dunsheath et al., in prep.). Similarly, in an analysis of various $\mathrm{HCN}, \mathrm{HCO}+$, and $\mathrm{CO}$ isotope transitions in the central $100 \mathrm{pc}$ of NGC 1068, Krips et al. (2011) argued in favour of warm $(T \gtrsim 200 \mathrm{~K})$ gas. However, they also concluded that the density is of the order of $n_{\mathrm{H}_{2}} \sim 10^{4} \mathrm{~cm}^{-3}$. Our LVG calculations indicate that such densities are associated with very high velocity gradients for the observed line ratio, which we consider physically unlikely. This, combined with a comparison of the cloud density to mean density estimated in Sect. 3.2 has led us to favour the higher density solution that has a more moderate velocity gradient.

\subsection{X-ray ionisation rate in NGC 3227}

Our LVG calculations presented above suggest that, as one alternative, the high $\mathrm{HCN}(1-0) / \mathrm{CO}(2-1)$ ratio in the central $\sim 100$ pc of NGC 3227 could result from an exceptionally high $\mathrm{HCN}$ abundance, an interpretation supported by the even higher $\mathrm{HCN} / \mathrm{CO}$ intensity ratios observed in the nuclei of NGC 1068 and NGC 6951. It is possible that the high $\mathrm{HCN}$ abundances are associated with elevated X-ray ionisation and/or heating rates near the AGN. Theoretical investigations of X-ray (or cosmicray) driven chemistry show that the (steady-state) molecular abundances depend primarily on the ratio of the cloud density $n$ 
to X-ray ionisation rate $\zeta$ (Krolik \& Kallman 1983; Lepp \& Dalgarno 1996; Maloney et al. 1996; Meijerink \& Spaans 2005; Boger \& Sternberg 2005; Meijerink et al. 2007). Here we adopt the notation of Boger \& Sternberg (2005), normalising $\zeta$ to $10^{-17} \mathrm{~s}^{-1}$ to give the parameter $n / \zeta_{-17}$. The density $n=n_{H}+n_{\mathrm{H}_{2}}$ refers to the total atomic plus molecular hydrogen density. In the following analysis, we first calculate the ratio $n / \zeta_{-17}$ in the nucleus of NGC 3227 and then compare it to ratios predicted by models of X-ray irradiated gas.

\subsection{1. $n / \zeta_{-17}$ in NGC 3227}

To estimate the X-ray ionisation rate in the central $\sim 100 \mathrm{pc}$ of NGC 3227 we need to know the intrinsic spectral energy distribution of the AGN. We adopt the SXPL model of Markowitz et al. (2009) in which both the hard and soft components of the $\mathrm{X}$-ray flux are modelled with power laws

$N_{\text {ph }}=0.0040(E / \mathrm{keV})^{-3.35}+0.0067(E / \mathrm{keV})^{-1.57}$,

where $E$ is the photon energy in $\mathrm{keV}$ and $N_{\mathrm{ph}}$ is the photon flux in units of $\mathrm{ph} \mathrm{keV}^{-1} \mathrm{~cm}^{-2} \mathrm{~s}^{-1}$. Making the usual assumption that the primary ionisation rate of hydrogen is negligible compared to the secondary ionisation rate, we then calculate the resulting ionisation rate $\zeta$ using equation A4 of Maloney et al. (1996)

$\zeta=N_{\mathrm{sec}} \int_{E_{\min }}^{E_{\max }} \sigma_{\mathrm{pa}}(E) F(E) \mathrm{d} E$.

Here $N_{\text {sec }}=28$ (as given by Maloney et al. 1996) is the number of secondary ionisations per $\mathrm{keV}$ of primary photoelectron energy, assuming a mean-energy per ion-pair of $37.1 \mathrm{eV}$ for energy deposition in a molecular hydrogen gas (Dalgarno et al. $1999) ; \sigma_{\mathrm{pa}}(E)$ is the absorption cross-section per $\mathrm{H}$ nucleus, for which we adopt the broken power-law fit in Eq. (A5) of Maloney et al. (1996); and $F(E)$ is the incident flux in units of $\mathrm{ph} \mathrm{keV}^{-1}$. The photoionisation is dominated by photons with energies for which $\tau \sim 1$. We therefore take the limits of the integral to be $E_{\max }=100 \mathrm{keV}$ and $E_{\min }$ as the energy at which the optical depth due to photoelectric absorption is $\tau(E)=1$, ignoring attenuation above this limit. The energy at which $\tau(E)=1$ is interpolated from Table 9.3 of Seward (2000) for the Morrison \& McCammon (1983) model, at a given column density. The column density is assumed to be proportional to distance from the AGN up to a maximum of $3 \times 10^{23} \mathrm{~cm}^{-2}$ (Hicks et al. 2009) at $30 \mathrm{pc}$.

We have evaluated the integral at two distances: $18 \mathrm{pc}$, an area weighted mean distance from the AGN to the HCN emitting gas that corresponds to the nuclear region; and $140 \mathrm{pc}$, corresponding to the distance of the circumnuclear ring. We find $\zeta_{18 \mathrm{pc}}=3.6 \times 10^{-13} \mathrm{~s}^{-1}$ and $\zeta_{140 \mathrm{pc}}=3.5 \times 10^{-15} \mathrm{~s}^{-1}$, about a factor of 100 smaller primarily due to the distance related geometrical dilution of the incident X-ray flux. The inferred ionisation rates are much higher than the typical ionisation rates in Galactic clouds.

Adopting a characteristic density $n_{\mathrm{H}_{2}} \sim 10^{5.5} \mathrm{~cm}^{-3}$ from our LVG analysis in Sect. 3.3. yields $n / \zeta_{-17} \sim 10$ in the nuclear region of NGC 3227. We note that even if the gas density were an order of magnitude higher (leading to proportionally higher $\left.n / \zeta_{-17}\right)$, the physical conditions would be well within the highionisation phase for the molecular chemistry.

\subsubsection{Models of abundance ratio as a function of $n / \zeta_{-17}$}

The model computations of Boger \& Sternberg (2005, 2006) show that gas can exist in a high (low) ionisation phase for small

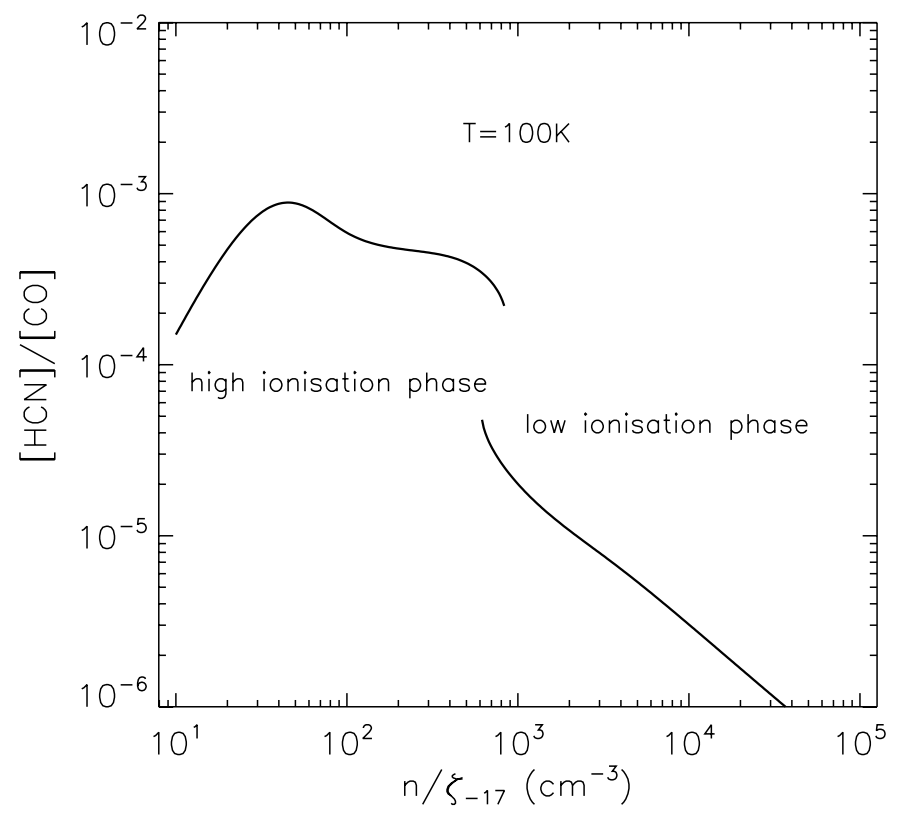

Fig. 4. Abundance ratio $[\mathrm{HCN}] /[\mathrm{CO}]$ as a function of $n / \zeta_{-17}$, the ratio of the local gas volume density to ionisation rate, for $100 \mathrm{~K}$ gas, showing the transition from the high- to low-ionisation phase.

(large) values of $n / \zeta_{-17}$, with a density ratio of $n / \zeta_{-17} \sim 10^{3}$ marking the cross-over between the two regimes. The HCN/CO abundance ratio can become large $\gtrsim 10^{-3}$ in the high-ionisation phase even at low gas temperatures, owing to the large densities of atomic and ionic carbon. Here we have re-run these calculations using the same elemental gas-phase abundances, and with a slightly updated reaction set. Figure 4 shows the resulting abundance ratios as a function of $n / \zeta_{-17}$ for $100 \mathrm{~K}$ gas. At very low $n / \zeta_{-17} \lesssim 100$, corresponding to that in the nuclear region of NGC 3227 , the HCN/CO abundance ratio approaches $\sim 10^{-3}$. For comparison, Lepp \& Dalgarno (1996) found a peak value of $5 \times 10^{-4}$ in their calculation. These models track the abundances of numerous molecules across many orders of magnitude and show that at low $n / \zeta_{-17}$, the HCN/CO abundance ratio is several orders of magnitude higher than that typically expected, to a level at which it approaches - and, given the uncertainties in these models, is commensurate with - that implied by the LVG models.

Harada et al. (2010) have shown that at elevated temperatures $\gtrsim 300 \mathrm{~K}$, rapid hydrogenation of $\mathrm{CN}$ to $\mathrm{HCN}$ can increase the $\mathrm{HCN} / \mathrm{CO}$ abundances yet further, and they comment that such warm gas may be present in the X-ray heated gas near AGN. We note, however, that their higher temperature models, for which $\mathrm{HCN} / \mathrm{CO}$ is largest, correspond to $n / \zeta_{-17}=10^{4.5}$. This is significantly larger than the value we are suggesting is characteristic for the nucleus of NGC 3227.

The models show that a combination of X-ray ionisation and heating do yield high $\mathrm{HCN}$ abundances, although not yet quite as high as the value of $\mathrm{HCN} / \mathrm{CO} \sim 10^{-2}$ implied by the LVG analysis in Sect. 3.3 for the nucleus. Further chemical modeling is required, but a high $\mathrm{HCN} / \mathrm{CO}$ intensity ratio in the nucleus resulting from elevated $\mathrm{X}$-ray ionisation rates appears plausible. In this picture, the lower $\mathrm{HCN} / \mathrm{CO}$ intensity ratio in the ring may simply reflect the lower ionisation rate there, and not just a lower gas density in this circumgalactic environment. 


\section{Summary and conclusions}

We have presented an LVG analysis of high-resolution observations of $\mathrm{CO}(2-1)$ and $\mathrm{HCN}(1-0)$ line emissions in the central regions of the Seyfert galaxy NGC 3227. We have found that

- The HCN(1-0)/CO(2-1) ratio in NGC 3227 is an order of magnitude higher in the central $80 \mathrm{pc}$ than in the circumnuclear ring at a radius of $140 \mathrm{pc}$. We note that NGC 6951 and NGC 1068 have similarly high published ratios in their central $\sim 100$ pc.

- The nuclear HCN/CO intensity ratio in NGC 3227 may be a result of optically thick line emission in dense gas, in which case there is only a weak constraint on the HCN/CO abundance ratio. However, our LVG calculations also indicate that the high nuclear ratios in all three of the galaxies are consistent with a single set of physical properties corresponding to warm $\sim 300 \mathrm{~K}$, dense $10^{5.5} \mathrm{~cm}^{-3}$ gas, in which the emission lines are optically thinner, but in which the $\mathrm{HCN} / \mathrm{CO}$ abundance ratio is very large $\sim 10^{-2}$. For these conditions the velocity gradients are $\mathrm{d} V / \mathrm{d} r \sim 100 \mathrm{~km} \mathrm{~s}^{-1}$, but would increase significantly at lower temperatures or densities. The clouds are most likely gravitationally unbound.

- The X-ray ionisation rate at radii less than $\sim 20$ pc may exceed $10^{-13} \mathrm{~s}^{-1}$, and could plausibly lead to high HCN abundances in molecular gas in the high-ionisation phase where the ratio of the gas density to the $\mathrm{X}$-ray ionisation rate is small.

Acknowledgements. The authors thank the IRAM staff, in particular Jeremie Boissier and Sascha Trippe, for their invaluable help in obtaining and reducing the data presented in this paper. We thank J. Graciá-Carpio for many useful and interesting discussions. We thank the DFG for support via German- Israeli Project Cooperation grant STE1869/1-1.GE625/15-1.

\section{References}

Boger, G., \& Sternberg, A. 2005, ApJ, 632, 302

Boger, G., \& Sternberg, A. 2006, ApJ, 645, 314

Dalgarno, A., Yan, M., \& Liu, W. 1999, ApJS, 125, 237

Davies, R. I., Thomas, J., Genzel, R., et al. 2006, ApJ, 646, 754

Davies, R., Müller Sánchez, F., Genzel, R., et al. 2007, ApJ, 671, 1388

Goldsmith, P. 2001, ApJ, 557, 736

Graciá Carpio, J., García Burillo, S., Planesas, P., \& Colina, L. 2006, ApJ, 640, L135

Graciá Carpio, J., García Burillo, S., Planesas, P., Fuente, A., \& Usero, A. 2008, A\&A, 479, 703

Green, S., \& Thaddeus, P. 1974, ApJ, 191, 653

Harada, N., Herbst, E., \& Wakelam, V. 2010, ApJ, 721, 1570

Hicks, E., Davies, R., Malkan, M., et al. 2009, ApJ, 696, 448

Kohno, K. 2005, in The Evolution of Starbursts, ed. S. Hüttermeister, E. Manthey, D. Bomans, \& K. Weis, AIP Conf. Proc., 783, 203

Kohno, K., Matsushita, S., Vila-Vilaró, B., et al. 2001, in The Central Kiloparsec of Starbursts and AGN, ed. J. Knapen, J. Beckman, I. Shlosman, \& T. Mahoney, ASP Conf. Proc., 249, 672

Kohno, K., Muraoka, K., Hatsukade, B., et al. 2008, in Far-Infrared Workshop, ed. C. Kramer, S. Aalto, \& R. Simon, EAS Publ. Ser., 31, 65

Krips, M., Neri, R., García-Burillo, S., et al. 2007, A\&A, 468, L63

Krips, M., Neri, R., Garciá Burillo, S., et al. 2008, ApJ, 677, 262

Krips, M., Martín, S., Eckart, A., et al. 2011, ApJ, 736, 37

Krolik, J., \& Kallman, T. 1983, ApJ, 267, 610

Lepp, S., \& Dalgarno, A. 1996, A\&A, 306, L21

Maloney, P., Hollenbach, D., \& Tielens, A. 1996, ApJ, 466, 561

Markowitz, A., Reeves, J., George, I., et al. 2009, ApJ, 691, 922

Meijerink, R., \& Spaans, M. 2005, A\&A, 436, 397

Meijerink, R., Spaans, M., \& Israel, F. 2007, A\&A, 461, 793

Morrison, R., \& McCammon, D. 1983, ApJ, 270, 119

Sani, E., et al. 2011, MNRAS, submitted

Schinnerer, E., Eckart, A., \& Tacconi, L. 2000, ApJ, 533, 826

Seward, F., in Allen's Astrohpysical Quantities, 4th edition, ed. A. Cox, 183

Sternberg, A., Genzel, R., \& Tacconi, L. 1994, ApJ, 436, L131

Tacconi, L. J., Genzel, R., Smail, I., et al. 2008, ApJ, 680, 246

Usero, A., Garciá-Burillo, S., Fuente, A., Martin-Pintado, J., \& RodríguezFernández, N. 2004, A\&A, 419, 897

Van der Tak, F., Black, J., Schöier, F., Jansen, D., \& van Dishoeck, E. 2007, A\&A, 468, 627

Yang, B., Stancil, P. C., Balakrishnan, N., \& Forrey, R. C. 2010, ApJ, 718, 1062 